\title{
Réalisation d'un simulateur de gestion d'énergie électrique embarquée
}

\author{
Y. AÏT-AMIRAT - H. GUALOUS \\ youcef.ait-amirat@univ-fcomte.fr, hamid.gualous@univ-fcomte.fr \\ Université de Franche-Comté - Département des Sciences Louis Néel \\ Rue Chantereine - BP 50547 - 90016 BELFORT Cedex
}

RESUME : L'objectif de l'article est la présentation d'un simulateur pour la gestion de l'énergie électrique embarquée à bord d'un véhicule. Ce simulateur se présente sous la forme d'une maquette pédagogique réalisée par des étudiants dans le cadre des projets du Master PROTEE (production et traitement de l'énergie électrique) de l'université de franche-comté. Il intègre des éléments issus de technologies récentes telles que l'alternodémareur qui équipe la Citroën C3, des supercondensateurs, un convertisseur d'énergie statique réversible (onduleur/redresseur), un volant d'inertie ainsi qu'une batterie classique. Ces divers éléments sont interconnectés entre eux de façon électrique ou mécanique. Le bon fonctionnement de l'ensemble a nécessité la réalisation de plusieurs cartes électroniques autour des microcontrôleurs PIC permettant l'exploitation dans un cadre pédagogique de la maquette. En effet, ces différentes cartes électroniques permettent de traiter les informations issues des différents capteurs, le contrôle du convertisseur statique, l'interface de dialogue avec l'utilisateur et un système informatique telle que dSPACE. Ces derniers sont introduits afin d'assurer, d'une part la supervision, la surveillance et la sûreté de fonctionnement de l'ensemble, et d'autre part, la mise en oeuvre de différentes stratégies de gestion de l'énergie. L'évaluation des différents rendements et puissances mécaniques et électriques mises en jeu est possible aussi bien en ligne qu'en différé puisque le système assure le stockage des différentes mesures. Ce projet est pluridisciplinaire et permet aux étudiants de faire le lien entre les différents modules enseignés en Master PROTEE.

\section{Mots clés : Conversion de l'énergie - Alternodémarreur - Supercondensateur - Volant d'inertie - DSpace.}

\section{1}

\section{INTRODUCTION}

Les récents progrès dans le domaine de la motorisation électrique et les dispositifs de stockage de l'énergie embarquée doivent permettre le développement des véhicules hybrides pour diminuer les émissions des polluants dues aux transports. Avec cette configuration le concept ZEV (Zero Emission Vehicle) peut être envisageable en milieu urbain. En effet, la motorisation électrique présente plusieurs avantages, l'absence d'émissions de polluant, des émissions sonores très réduites, un couple au démarrage élevé ce qui rend la conduite urbaine particulièrement agréable. L'autonomie étant l'élément discordant à ce tableau, l'alimentation électrique doit présenter un élément de stockage avec une densité massique d'énergie et de puissance élevée comme l'association des batteries et des supercondensateurs ou à plus long terme l'association pile à combustible supercondensateurs.

Plusieurs auteurs s'accordent pour dire que pour un véhicule d'environ $1300 \mathrm{~kg}$, suivant la fonction réalisée et en choisissant une alimentation électrique appropriée (batterie + supercondensateurs par exemple) on peut réduire les émissions de $\mathrm{CO}_{2}$ comme le montre le tableau 1 ci-dessous [1-3].

L'exemple incontournable de véhicule hybride commercialisé est la Toyota Prius qui a montré qu'il est possible de réduire les émissions tout en gardant un coût raisonnable. D'autres véhicules hybrides ou à pile à combustible ont été réalisés, cependant les problèmes de la récupération de l'énergie et l'optimisation énergétique à bord restent à résoudre.

\begin{tabular}{|c|c|c|}
\hline Fonctions & $\begin{array}{c}\text { Puissance du } \\
\text { moteur électrique }\end{array}$ & $\begin{array}{c}\text { Gain en } \\
\text { émission de } \\
\mathrm{CO}_{2}\end{array}$ \\
\hline $\begin{array}{l}\text { Arrêt du moteur } \\
\text { thermique au ralenti } \\
+ \text { récupération de } \\
\text { l'énergie au freinage }\end{array}$ & De l'ordre de5 kW & $\begin{array}{c}\text { Environ } 10 \\
\%\end{array}$ \\
\hline $\begin{array}{l}\text { Arrêt du moteur } \\
\text { thermique au ralenti } \\
+ \text { récupération de } \\
\text { l'énergie au freinage } \\
+ \text { sous dimension- } \\
\text { nement du moteur } \\
\text { thermique et assis- } \\
\text { tance en accéléra- } \\
\text { tion }\end{array}$ & De l'ordre $10 \mathrm{~kW}$ & $\begin{array}{c}\text { Environ } 20 \\
\% \\
\text { à } 30 \%\end{array}$ \\
\hline $\begin{array}{l}\text { Arrêt du moteur } \\
\text { thermique au ralenti } \\
+ \text { récupération de } \\
\text { l'énergie au freinage } \\
+ \text { downsizing du } \\
\text { moteur thermique et } \\
\text { assistance en accélé- } \\
\text { ration + hybride } \\
\text { total }\end{array}$ & De l'ordre $30 \mathrm{~kW}$ & $\begin{array}{c}\text { Environ } 40 \\
\% \\
\text { à } 45 \%\end{array}$ \\
\hline
\end{tabular}

Tableau 1. Estimation (en \%) des gains en émission $\mathrm{CO}_{2}$

D'autres prototypes utilisant des supercondensateurs ont été réalisés et sont en exploitation en Europe, au Japon et en Amérique du nord. Parmi ces exemples, 
on peut citer le poids lourd de Nissan qui utilise des supercondensateurs. Ces derniers ont une masse de 194 $\mathrm{kg}$ et une énergie de $1310 \mathrm{Wh}$. Nissan annonce $50 \%$ de réduction de consommation par rapport à un diesel classique. Ce véhicule est commercialisé. Un autre type d'hybridation a montré l'apport des supercondensateurs, il s'agit des véhicules à pile à combustible. Même si les spécialistes annoncent que ce véhicule ne sera attractif qu'en 2030 à cause du manque de maturité de la technologie des piles à combustible et des infrastructures nécessaires. Parmi les démonstrateurs réalisés, il y a le véhicule HY-LIGHT de Michelin et PSI [4]. C'est un véhicule zéro émission qui utilise une pile à combustible à électrolyte polymère et des supercondensateurs. Ces derniers fournissent une puissance de 32 à 45 $\mathrm{kW}$ pendant 15 à 20s.

Si l'hybridation de la motorisation et des sources de stockage de l'énergie constitue une solution incontestable aujourd'hui pour réduire la pollution liée aux émissions de polluants, la commande et la gestion de l'énergie déterminent en grande partie les performances du véhicule. Ainsi une gestion intelligente de l'énergie entre les différentes sources permet d'optimiser la consommation énergétique du véhicule et donc d'augmenter le rendement tout en diminuant la consommation en carburant du véhicule.

L'intégration des supercondensateurs dans un système multi-sources tel que le véhicule hybride constitue par excellence un dispositif où les contraintes pour la gestion de l'énergie sont les plus sévères. En effet, pour augmenter le rendement du système et diminuer la consommation du véhicule, il faut récupérer le maximum possible de l'énergie lors du freinage. Les supercondensateurs doivent être utilisés systématiquement pour assister les accélérations du véhicule et lors des phases transitoires liées à la consommation des auxiliaires du véhicule.

Dans un premier temps, nous avons considéré une alimentation associant une batterie et un module de supercondensateurs. Cependant des études sont en cours pour la récupération de l'énergie à l'aide des supercondensateurs. La démarche que nous avons adoptée consiste à remplacer le freinage du véhicule par un volant d'inertie avec un facteur d'échelle au niveau de la puissance. Le dispositif consiste à relier à l'alimentation hybride un onduleur qui alimente un moteur électrique. Ce dernier entraîne un volant d'inertie qui, une fois sa vitesse nominale atteinte on inverse le sens du flux de puissance. Le moteur fonctionne alors en génératrice et permet de récupérer de l'énergie pour charger les supercondensateurs.

En plus de la commande rapprochée qu'il faut développer pour les convertisseurs, il est primordial d'établir une loi de gestion de l'énergie en tenant compte des différentes contraintes.
Nous proposons dans la section 2 de décrire l'ensemble des composants de la maquette mise en œuvre et dans la section 3, nous explorons les possibilités d'exploitation de la maquette dans différentes disciplines de l'EEA à savoir l'automatique, l'informatique industrielle, l'électronique et l'électrotechnique. La conclusion est faite sur le coût et la reproductibilité d'une telle maquette.

\section{DESCRIPTION DES ELEMENTS DU SI- MULATEUR DU VEHICULE}

\subsection{Alternodémarreur}

L'alterno-démarreur (fig. 1) a été introduit dans la voiture pour assurer la fonction «Stop\&Go » qui consiste à couper le moteur thermique lorsque le véhicule est à l'arrêt et de le redémarrer instantanément quand le conducteur enclenche une vitesse ou relâche le frein (pour les boîtes automatiques). L'impact est direct sur la consommation urbaine dont la réduction annoncée est estimée de 5 à $10 \%$.

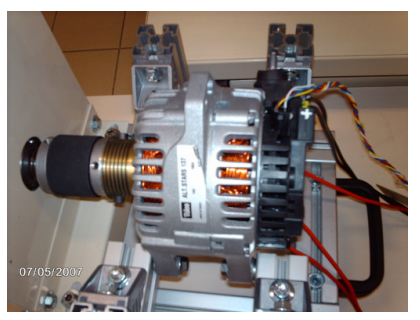

fig 1 : Alternodémarreur

Il s'agit d'une machine synchrone à rotor bobiné à excitation séparée. En mode moteur, elle assure le démarrage du moteur thermique. Ses caractéristiques dans ce cas sont :

Puissance de démarrage $2,1 \mathrm{~kW}$;

Couple au démarrage $65 \mathrm{Nm}$

En mode alternateur, cette machine permet de maintenir la charge des batteries et assurer l'alimentation des équipements auxiliaires embarqués de plus en plus nombreux. Ses caractéristiques sont :

Puissance au régime de ralenti $2,0 \mathrm{~kW}$;

Puissance maximale $2,7 \mathrm{~kW}$;

Rendement maximal $80 \%$

Sa tension de travail est de $14 \mathrm{~V}$.

Dans la figure 2, un schéma de l'alimentation électrique du système est présenté. Nous distinguons le hacheur alimentant l'excitation. En mode moteur le rapport cyclique est de 1 et l'excitation se fait alors avec la valeur nominale du courant. En mode alternateur, le rapport cyclique variable permet de contrôler le courant d'excitation en fonction du courant de recharge de la batterie et/ou des supercondensateurs. Sur les figures 3 et 4 , nous explicitons le schéma de notre alimentation électrique de l'alternodémareur dans les 2 cas de fonctionnement : moteur et générateur. 


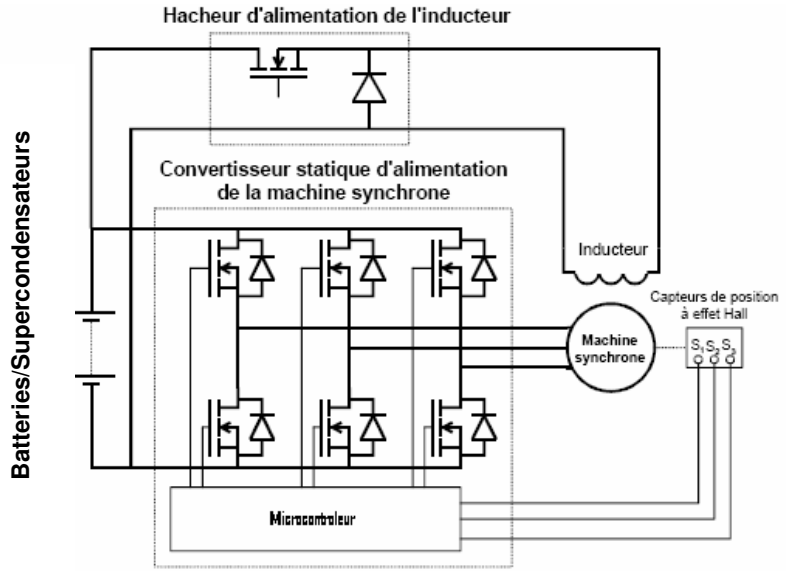

fig 2 : Schéma de l'alimentation électrique

\subsubsection{Fonctionnement en moteur}

Le système de la figure 2 peut se ramener dans ce mode de fonctionnement au schéma de la figure suivante :

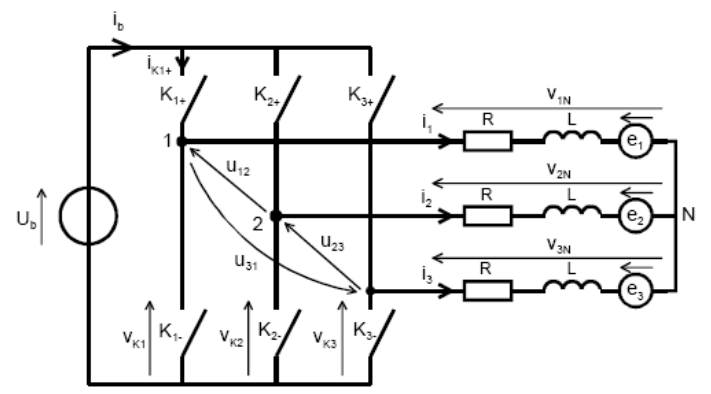

fig 3 : Fonctionnement en onduleur

Compte tenu du fait que notre machine synchrone présente une fem trapézoïdale, nous avons opté pour une alimentation avec des créneaux de courant de $120^{\circ}$ comme indiqué sur la photo prise de l'oscilloscope pendant les essais :

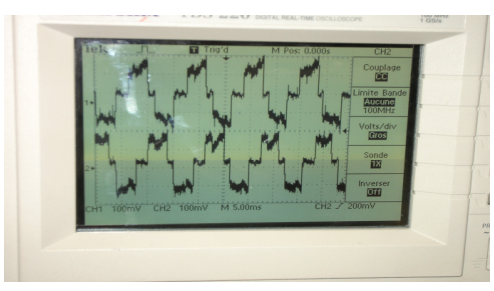

fig 4 : Courants dans 2 phases du moteur

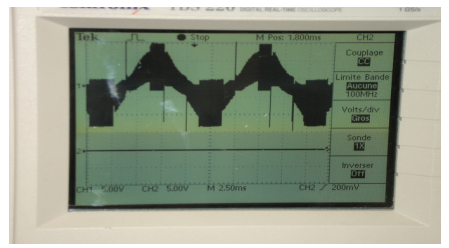

fig 5 : Tension entre 2 phases du moteur
Par ailleurs un relevé simultané des tensions induites à vide et des 3 signaux issus du capteur à effets Hall (capteurs 6 positions) nous a permis d'établir une loi permettant de générer les 6 signaux commandant les différents transistors, à savoir :

$$
\begin{gathered}
K_{1+}=S_{1} \cdot \overline{S_{2}} ; K_{2+}=S_{2} \cdot \overline{S_{3}} ; K_{3+}=S_{3} \cdot \overline{S_{1}} \\
K_{1-}=\overline{S_{1}} \cdot S_{2} ; K_{2-}=\overline{S_{2}} \cdot S_{3} ; K_{3-}=\overline{S_{3}} \cdot S_{1}
\end{gathered}
$$

où : $K_{\mathrm{i}+}$ représente la commande du transistor relié au potentiel le plus haut du bus d'alimentation

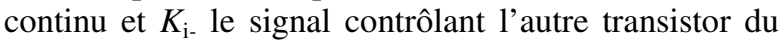
même bras i (i=1, 2 ou 3) comme indiqué sur la figure 3. $S_{1}, S_{2}$ et $S_{3}$ sont les 3 signaux issus de notre capteur à 6 positions (voir figure 2). Il s'agit de grandeurs prenant des valeurs logiques (0 ou 1$)$. A noter que pour réaliser cet essai, nous avons utilisé une autre machine pour entraîner notre alternodémarreur.

\subsubsection{Fonctionnement en générateur}

Dans ce cas, le contrôle des transistors est complètement inhibé. Seules interviennent les diodes mises en antiparallèle comme indiquées sur la figure 6.

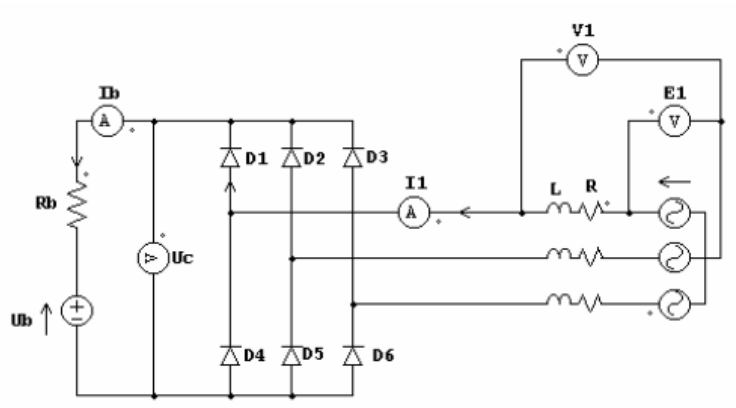

fig 6 : Fonctionnement en redresseur

Le courant débité par le redresseur pourrait être contrôlé par le courant (ou la tension) d'excitation ou par la vitesse d'entraînement de l'alternateur. Sur les véhicules, on opte plutôt sur la deuxième solution qui permet de dimensionner une alimentation constante facile à mettre en œuvre et d'agir sur l'injection du moteur thermique qui entraîne l'alternateur, donc si la vitesse augmente, l'amplitude maximum de la tension induite augmente aussi.

S'agissant de notre maquette, l'entraînement de l'alternodémarreur est assuré par le volant d'inertie dont la vitesse est variable mais non contrôlable (elle est l'image même de la capacité d'énergie cinétique stockée) qui aura donc tendance à diminuer. Nous allons introduire un hacheur permettant le contrôle du courant d'excitation. Celui-ci sera faible quand la vitesse du volant d'inertie est à son maximum et augmentera au fur et à mesure que la vitesse diminue. Cette étude fera l'objet d'un projet et pourrait être exploitée dans le cadre de travaux pratiques mettant en œuvre le contrôle non linéaire d'un alternateur à vitesse variable. 


\subsection{Supercondensateur}

Le supercondensateur possède une puissance instantanée plus importante que celle des batteries et une énergie plus grande que celle des condensateurs [7]. Sa durée de vie est plus élevée que celle des batteries (environ 10 ans). Le diagramme de Ragone (figure 7) représente les différents systèmes de stockage d'énergie dans le plan puissance spécifique/énergie spécifique. Ce diagramme montre que les condensateurs électrochimiques possèdent une très grande densité de puissance mais une très faible énergie spécifique. Ils sont utilisés généralement pour des constantes de temps inférieures à quelques centaine de ms.

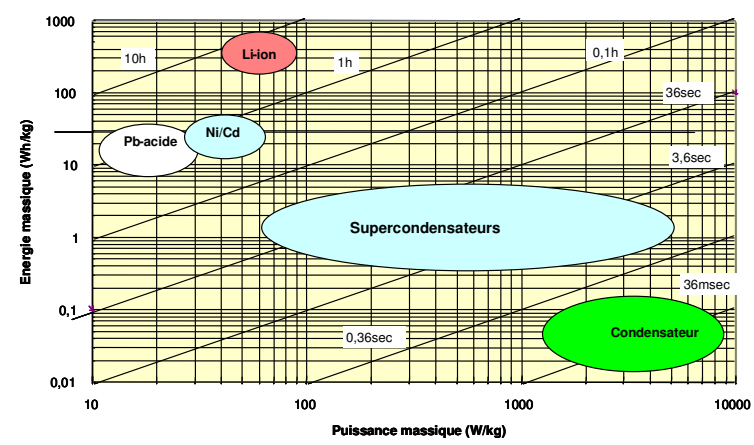

fig 7 : Diagramme de Ragone

Les batteries ont une densité de puissance très faible et une énergie spécifique élevée. Elles peuvent être utilisées avec une constante de temps supérieure à la minute. C'est entre les batteries et les condensateurs électrochimiques que se situent les supercondensateurs qui sont utilisés le stockage de l'énergie avec une constante de temps inférieure à quelques dizaines de secondes. Le tableau suivant résume les performances des différents éléments de stockage évoqués ci-dessus.

\begin{tabular}{|c|c|c|c|}
\hline & $\begin{array}{l}\text { Condensateur } \\
\text { électrolytique }\end{array}$ & $\begin{array}{c}\text { Supercondensa- } \\
\text { teur }\end{array}$ & Batterie \\
\hline $\begin{array}{l}\text { Temps de } \\
\text { charge t }\end{array}$ & $\mu \mathrm{s}<\mathrm{t}<\mathrm{ms}$ & $1 \mathrm{~s}<\mathrm{t}<30 \mathrm{~s}$ & $1 \mathrm{~h}<\mathrm{t}<5 \mathrm{~h}$ \\
\hline $\begin{array}{l}\text { Temps de dé- } \\
\text { charge }\end{array}$ & $\mu \mathrm{s}<\mathrm{t}<\mathrm{ms}$ & $1 \mathrm{~s}<\mathrm{t}<30 \mathrm{~s}$ & $0.3 h<3 h$ \\
\hline $\begin{array}{l}\text { Rendement } \\
\text { charge/décharg } \\
\text { e }\end{array}$ & $>95 \%$ & $\begin{array}{l}\text { entre } 85 \% \text { et } \\
98 \%\end{array}$ & $\begin{array}{l}\text { Entre } \\
70 \% \text { et } \\
85 \%\end{array}$ \\
\hline $\begin{array}{l}\text { Densité de } \\
\text { puissance } \\
(\mathrm{W} / \mathrm{kg})\end{array}$ & $>10^{6}$ & $10^{4}$ & $<10^{3}$ \\
\hline $\begin{array}{l}\text { Densité } \\
\text { d'énergie } \\
(\mathrm{Wh} / \mathrm{kg})\end{array}$ & $\begin{array}{l}\text { entre } 10 \text { et } \\
100\end{array}$ & entre 1 et 10 & $\begin{array}{l}\text { Entre } 10 \text { et } \\
100\end{array}$ \\
\hline $\begin{array}{l}\text { Durée de vie } \\
\text { nombre de } \\
\text { cycles }\end{array}$ & $10^{10}$ & $10^{6}$ & $10^{3}$ \\
\hline
\end{tabular}

Tableau 2 : Comparaison entre quelques éléments de stockage de l'énergie

D'après les données du tableau 2, il est clair que le supercondensateur est un élément potentiel pour le stockage tampon de l'énergie électrique embarquée. Il peut être utilisé comme une source pour les demandes de fortes puissances jusqu'à quelques dizaines de se- condes. Notons que le nombre de cycles de charge/décharge supportés un supercondensateur est environ 1000 fois plus élevé que celui d'une batterie. De plus, le supercondensateur peut fournir ou absorber un courant très élevé, ceci est très important pour la récupération de l'énergie lors du freinage par exemple. Les supercondensateurs présentent une résistance série plus faible que les batteries. Le rendement de charge/décharge d'un supercondensateur est supérieur à $95 \%$ alors que dans le cas d'une batterie il est compris entre 70 et $85 \%$.

Nous proposons sur la figure 8 , un hacheur réversible permettant de connecter nos supercondensateurs au bus continu, dont la tension est imposée par la batterie. La charge des supercondensateurs est assurée aussi bien par la batterie que par l'alternodémarreur (en mode alternateur). L'énergie ainsi stockée servira d'appoint pour soulager la batterie lors des phases successives de démarrage ou lors de l'entraînement du volant d'inertie sur notre maquette.

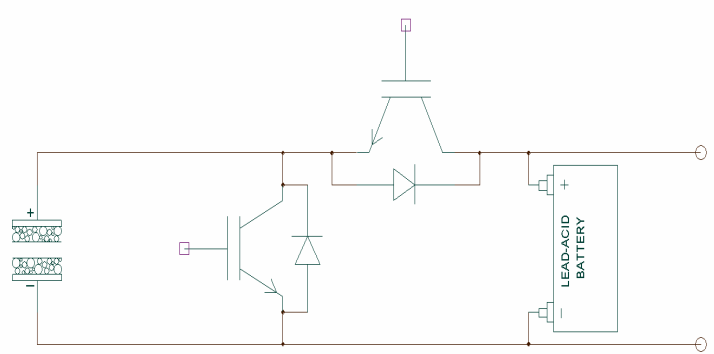

fig 8: Interfaçage supercondensateurs/batterie

\subsection{Volant d'Inertie}

Les volants d'inertie, que l'on appelle aussi batteries électromécaniques, permettent de stocker l'énergie sous forme cinétique $\left(1 / 2 J N^{2}\right)$, avec $J$ le moment d'inertie et $N$ la vitesse.

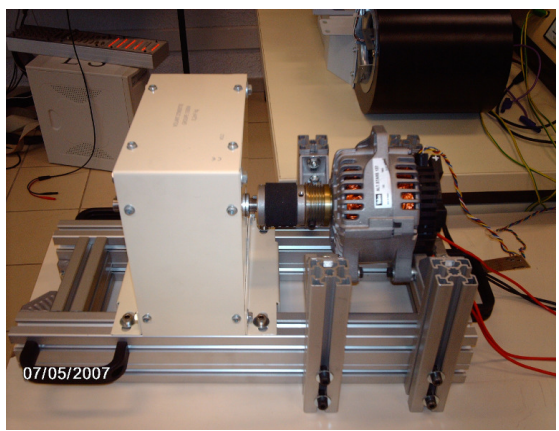

fig 9 : Volant d'Inertie

Ils permettent donc de convertir l'énergie électrique sous forme cinétique pour ensuite effectuer la conversion inverse lorsqu'il y a besoin. Cette double transformation électromécanique nécessite un moteur pour entraîner le volant d'inertie. Ce dernier est ensuite découplé du moteur et conserve l'énergie dans sa rotation. Lorsqu'il y a une demande de puissance ou 
d'énergie, le volant d'inertie entraîne le moteur qui fonctionne alors en génératrice et fournit de l'énergie électrique. Ce principe est utilisé pour des applications stationnaires. Des recherches sont effectuées dans ce domaine pour des applications transport terrestre. Les performances en termes de densité de puissance et d'énergie stockée, dépendent en grande partie des matériaux utilisés pour la fabrication du dispositif luimême mais aussi des performances électriques du moteur réversible.

\section{EXPLOITATION PEDAGOGIQUE}

\subsection{Traitement de l'information}

\subsubsection{Autopilotage du moteur synchrone}

Il s'agit de générer les signaux de commandes des six transistors de notre onduleur à partir de l'acquisition des 3 signaux issus du capteur de position à effet Hall en suivant les relations données en (1). Pour la mise en œuvre, l'étudiant doit :

Repérer les 3 entrées de son microcontrôleur pour y relier les 3 signaux issus du capteur à effet Hall.

- Repérer les 6 sorties numériques aux différents circuits qui pilotent les transistors.

Programmer les relations données en (1).

Exécuter son programme.

On doit veiller à ce que les niveaux de tension des signaux numériques échangés avec le microcontrôleur (aussi bien en entrée qu'en sortie) soient adaptés.

\subsubsection{Contrôle du courant}

L'autopilotage du moteur étant assuré, la variation de sa vitesse peut se faire par la variation de la tension d'alimentation ou par le contrôle du courant. En complément à la première précédente, nous ajoutons un module permettant le contrôle du courant d'induit. Compte tenu du type d'alimentation retenue (créneau de $120^{\circ}$ ), le seul contrôle du courant en entrée de l'onduleur (courant continu) suffit. Sa mise en oeuvre peut se faire de 2 façons différentes :

a) solution numérique

La mesure du courant absorbé par l'onduleur (c'est-à-dire, coté continu) est faite à l'aide d'un capteur de courant à effet Hall $I_{\text {mes. }}$. Cette valeur est ensuite échantillonnée à l'aide du convertisseur analogique/numérique du microcontrôleur. La valeur binaire numérique ainsi obtenue est comparée à une valeur de référence pour ce courant donnée dans la même échelle (binaire). Cette différence $\left(I_{\mathrm{ref}}-I_{\mathrm{mes}}\right)$, notée $\Delta I$, doit rester dans une bande d'hystérésis $\varepsilon$. Pour cela, on détermine à chaque fois :

Si $\varepsilon>0$ alors $C=1$ sinon $C=0$

$$
K_{1+}=C \cdot S_{1} \cdot \overline{S_{2}} ; K_{2+}=C \cdot S_{2} \cdot \overline{S_{3}} ; K_{3+}=C \cdot S_{3} \cdot \overline{S_{1}}
$$

A noter que dans ce cas, l'hystérésis n'agit que sur l'un des deux interrupteurs qui sont censés conduire. La continuité du courant étant assuré par la diode anti-parallèle. Mais d'autres solutions sont envisageables.

b) solution analogique

Le même principe est réalisé sauf que nous mettrons en ouvre ici une solution analogique complète où la consigne du courant est une référence analogique (qui peut être piloté par le microcontrôleur associé à un convertisseur numérique analogique). Cette référence de courant $I_{\text {ref }}$ ainsi que la mesure de courant $I_{\text {mes }}$ attaquent un comparateur qui délivre un signal logique égale à 1 (état haut si, par exemple $I_{\mathrm{ref}} I_{\mathrm{mes}}$ ). Ce signal logique est ensuite démultiplié 3 (ou 6) fois pour être associé à chacun des 3 (ou 6) signaux de commandes issus du microcontrôleur.

L'objectif ici est d'analyser les avantages et les inconvénients de chacune des solutions mises en œuvre.

\subsubsection{Estimation de la vitesse}

Il s'agit ici d'associer l'un des signaux du capteur à effet Hall au fonctionnement du Timer. Nous pouvons régler par programmation la fréquence de décomptage du Timer que l'on note $f_{c}$. Dès que l'on détecte un front montant, on relève $\mathrm{C}_{1}$, au front montant suivant nous relevons $\mathrm{C}_{2}$.

En supposant que l'on tourne assez vite pour que le Timer ne soit pas repassé par zéro, nous pouvons estimer que la vitesse est donnée par :

$$
N=\frac{f_{c}}{\left(C_{1}-C_{2}\right)}
$$

La combinaison des 3 signaux S1, S2 et S3 nous permettrait d'estimer une vitesse encore plus faible que.

\subsection{Automatique}

\subsubsection{Régulation de vitesse (stockage inertielle)}

L'estimation de la vitesse $N$ de rotation comparée à une vitesse de référence voulue $N^{*}$ nous permet de générer automatiquement (à travers un régulateur numérique PI, par exemple) la consigne du courant $I_{\text {ref }}$ nécessaire à la génération de notre couple d'entraînement du volant d'inertie. Une fois cette vitesse de référence $N^{*}$ atteinte, le courant permettant de la maintenir sera nécessairement faible et nous renseignera sur les pertes mécaniques dans le système.

\subsubsection{Contrôle du courant de charge des super- condensateurs (stockage électrochimique)}

L'estimation de la vitesse $N$ de rotation du volant d'inertie nous permet de déterminer le courant (ou la tension) d'excitation nécessaire à notre alternodé- 
marreur de façon à pouvoir contrôler le courant de charge des supercondensateurs.

\subsection{Gestion et stockage de l'énergie}

L'étude de cette partie dont la mise en oeuvre est évoquée dans [5] et l'intérêt dans [6] est en cours de finalisation toujours dans le cadre d'un projet de dernière année du Master. Elle nécessite la mise en place d'un système informatique d'acquisition et de contrôle tels que dSPACE. Il faudrait disposer au sein du logiciel de tous les différents paramètres physiques du système (tension supercondensateurs, tension du bus continu, courant des supercondensateurs, courant à l'entrée de l'onduleur, tension entre phases de l'alternodémarreur et l'estimation de la vitesse du volant d'inertie). Grâce au programme développé pour cette application nous pouvons, par exemple, réaliser le travail suivant décliné en quatre points :

1) Déterminer l'état de charge initiale du module de supercondensateurs. Il s'agit de mesurer la tension du module de supercondensateurs et de calculer son état de charge appelé SOC, il caractérise la quantité d'énergie présente dans le module de supercondensateurs. Il vaut 1 lorsque le module est complètement chargé et 0 lorsqu'il est chargé à sa valeur minimale :

$$
S O C=\frac{4}{3}\left(\frac{E}{E_{\max }}-\frac{1}{4}\right)
$$

avec $E$ est l'énergie stockée définie à chaque instant par

$$
E=\frac{1}{2} C . V^{2}
$$

où $C$ est la capacité totale équivalente du bloc de supercondensateurs et $V$ la tension instantanée mesurée à ses bornes. $E_{\max }$ est l'énergie maximum emmagasinée, obtenue pour $V_{\max }$.

2) Entraîner le volant d'inertie jusqu'à une certaine vitesse $N^{*}$ à l'aide d'un régulateur (voir \&3.2.1). Estimer l'énergie cinétique stockée par le volant d'inertie en utilisant la formule donnée précédemment. La détermination du moment d'inertie $J$ pourrait faire l'objet d'un TP de mécanique. Pour notre part, on se réfère à la valeur donnée par le constructeur.

3) L'énergie cinétique emmagasinée par le volant d'inertie étant connue ainsi que l'état de charge des supercondensateurs (voir les points 1 et 2 précédents), on pourrait couper l'alimentation de l'alternodémarreur et brancher les supercondensateurs et procéder au transfert de l'énergie depuis le volant d'inertie jusqu'aux supercondensateurs moyennant le contrôle du courant de charge de ceux-ci (voir \&3.2.2).

4) Analyser les données expérimentales recueillies, faire le calcul des différentes puissances mises en jeu ; estimer le rendement global ainsi que chacun des éléments suivant les possibilités offertes.

\section{CONCLUSION}

Le travail autour de cette maquette continuera dans le cadre de projets dont l'objectif serait d'affiner les différentes régulations, régler quelques problèmes sur les cartes électroniques réalisées pour les différentes interfaces et enfin améliorer le dialogue entre le microcontrôleur PIC, chargé de la commande rapprochée du convertisseur associé à l'alternodémarreur et le système dSPACE. Le coût actuel de la maquette est estimé à plus de 3000 Euros avec une grosse part pour le convertisseur de l'alternodémarreur qui est surdimensionné. Nous préconisons de réduire la puissance de tous les éléments à une centaine de Watts, ce qui permettrait sans doute de réduire le coût de la maquette et de généraliser son usage dans des travaux pratiques pluridisciplinaires.

Rappelons que cette maquette qui est un simulateur pratique mettant en oeuvre le principe de la récupération de l'énergie cinétique lors du freinage du véhicule. Nous avons illustré les connaissances utilisées issues de l'informatique industrielle, automatique, électronique et une application d'un système informatique pour la supervision, la surveillance et le traitement des données.

\section{REFERENCES}

[1] A. Schneuwly, R. Smith 'Ultracapacitor to address power and redendancy needs of vehicles' EVS21, Monaco 2-6 April 2005, CDROM.

[2] P. Pinchon 'Futures évolutions des motorisations dans l'automobile' L'automobile du futur : les technologies de l'IFP - 6 mai 2004.

[3] Gabriel Plassat 'Les technologies des moteurs de véhicules lourds et leurs carburants' rapport ADEME Tome1, Département Technologies des transports, Ressource électronique 2006.

[4] P. Dietrich, D. Laurent, G. Paganelli, A. Tsukada, D. Walser, P. A. Magne, F. Büchi, P. Varenne, R. Kötz, S. Feunberger, A. Delfino, D. Olsommer 'Concept, design and first results of Light-WeightFuel-Cell vehicle 'HY-LIGHT', EVS-21, Monaco 2-6 April 2005. CDROM.

[5] A. Rufer - La conversion d'énergie électrique pour le stockage.

http://leiwww.epfl.ch/publications/rufer_etg_99_la usanne.pdf

[6] B. Multon - Stockage de l'énergie dans les applications stationnaires. Colloque Energies Electriques : besoins, enjeux, technologies et applications. Belfort, JUIN 2004.

[7] H. Gualous, R. Gallay 'Applications des supercondensateurs' D3335 Techniques de l'Ingénieur, AOUT 2007. 